\title{
Multi-walled carbon nanotube-based systems for improving the controlled release of insoluble drug dipyridamole
}

\author{
WENQUAN ZHU, HAITAO HUANG, YAN DONG, CUIYAN HAN, XIAOYU SUI and BAIYU JIAN \\ College of Pharmacy, Qiqihar Medical University, Qiqihar, Heilongjiang 161006, P.R. China
}

Received August 16, 2018; Accepted April 8, 2019

DOI: $10.3892 /$ etm.2019.7510

\begin{abstract}
Applicability of multi-walled carbon nanotubes (MWCNTs) in loading dipyridamole (DDM), a poorly soluble drug, was evaluated. Additionally, the effect of drug-loading efficiency on the release behavior of the MWCNT-DDM system was also investigated. DDM as a model drug was incorporated into MWCNTs with different drug-loading rates $(10,25$ and $50 \%)$ using the solvent deposition method. The MWCNT-DDM system was successfully established and characterized using common solid-state characterization methods. Scanning electron microscopy (SEM), transmission electron microscopy (TEM), nitrogen adsorption analysis and Fourier transform-infrared (FT-IR) spectroscopy were carried out to observe the progress of drug loading. X-ray diffraction (XRD) and differential scanning calorimetry (DSC) were used to systematically assess the crystalline state of the DDM after being loaded into the MWCNTs. Improvements in dissolution rate were evaluated by the dissolution test. The results revealed that with the increase of drug loading, the form of DDM in the MWCNTs changed from amorphous to crystalline state. Also, the release rate of DDM decreased upon increasing the drug-loading rate of carriers. In conclusion, MWCNTs are proven to be promising carriers for loading DDM.
\end{abstract}

\section{Introduction}

With the development of new carrier technologies, the use of carbon and carbon-related materials has gained the attention of numerous pharmaceutical researchers (1-5). Carbon materials include active carbon, multi-walled carbon nanotubes (MWCNTs) and fullerenes (6-10). Compared to traditional drug carriers, carbon materials possess several unique properties, which render them especially suitable for pharmaceutical research. For example, it is well recognized

Correspondence to: Dr Wenquan Zhu, College of Pharmacy, Qiqihar Medical University, 333 Bukui North Street, Qiqihar, Heilongjiang 161006, P.R. China

E-mail: zhuwenquan1984@126.com

Key words: dipyridamole, multi-walled carbon nanotube, drug delivery system, drug loading that active carbon has a strong adsorption, which enables it to have high loading capacity. Also, carbon nanotubes have remarkable advantages due to their nanoscale channel structure, stability, adsorption property and surface functionalization $(11,12)$, which make them suitable materials for drug delivery systems. Carbon nanotubes are widely used as drug carriers in the process of loading drugs (13). MWCNTs have been successfully used as drug delivery systems for loading Doxorubicin, Dorzolamide and Carbamazepine (14).

Dipyridamole (DDM) is a non-nitrate coronary vasodilator that inhibits platelet aggregation and can be used in combination with other anticoagulant drugs, such as warfarin, to prevent thrombosis in patients with valvular or vascular disease. DDM is also used in myocardial perfusion imaging, and as an antiplatelet agent, DDM's application, in combination with aspirin, has been reported in stroke prophylaxis $(15,16)$. DDM belongs to class II of the Biopharmaceutical Classification System (BCS), and it is poorly soluble in water. Thus, the dissolution process limits its rate and degree of oral absorption. In the present study, DDM was selected as a model drug in an attempt to improve its oral bioavailability by enhancing its solubility. In the past, various carriers have been employed to solve the solubility problem of DDM, such as liposomes, solid dispersion, and nanospheres (17-20). Unfortunately, there are very few carriers which possess advantages, such as high drug loading, system stability and drug release controllability. Therefore, identifying a suitable carrier for DDM is the research focus of the current study. Although MWCNTs have been recently used for loading other drugs, they have not been studied as drug delivery systems for DDM (21-23). Multiple studies have proposed carriers, including MWCNTs, with delineation of pore volume, surface area and surface modification (24-26). Among the many factors that play a role in the process of drug delivery, drug-loading efficiency is particularly critical for MWCNTs. In order to characterize the relationship between drug-loading efficiency and drug release, it is necessary to perform a systematic study to explore the ability of MWCNTs with different drug loading rates in enhancing the dissolution rate of poorly water-soluble drugs.

The current study evaluated the suitability of MWCNTs as carriers for DDM and elucidated whether they can improve the water solubility of DDM. DDM was incorporated into MWCNTs using the solvent deposition method at different ratios $(10,25$ and $50 \%)(27,28)$. The obtained samples were examined by scanning electron microscopy (SEM), transmission electron microscopy (TEM), Fourier transform-infrared (FT-IR) 
spectroscopy, X-ray diffraction (XRD) and differential scanning calorimetry (DSC). Subsequently, the behavior of DDM released from MWCNT with different drug-loading rates was compared and analyzed.

\section{Materials and methods}

Materials. DDM (purity $>99.0 \%$ ) was purchased from Wuhan Yuan Cheng Gong Chuang Technology Co., Ltd. (Wuhan, China). MWCNTs were purchased from Nanjing Xianfeng Nano Material Technology Co., Ltd. (Nanjing, China). All other chemical reagents were used in accordance with the requirements of analytical/HPLC grade.

Synthesis of MWCNT-DDM system. DDM was incorporated into MWCNTs at different percentages (10,25 and 50\%) using the solvent deposition method, which included soaking equilibrium and solvent evaporation. Briefly, DDM was dissolved in methanol to obtain a concentrated solution $(5 \mathrm{mg} / \mathrm{ml})$. Next, different amounts of MWCNTs were added to the drug solution to obtain a mixture. The ratio of drug/MWCNTs in the loading solution was 10,25 and $50 \%$, respectively. Then, the mixture was gently stirred for $24 \mathrm{~h}$ at room temperature in a closed container to complete the adsorption equilibrium operation. Finally, the products were dried at $40^{\circ} \mathrm{C}$ in air to remove the organic solvent. Drug-loaded samples were labeled DM-10, DM-25 and DM-50, respectively.

\section{Characterization techniques}

SEM observation. A field emission scanning electron microscope (Hitachi S-4300; Hitachi, Ltd., Tokyo, Japan) was employed to characterize the morphology of the obtained samples (20). A small number of samples were fixed on metal stubs and sputtered with gold under vacuum conditions.

TEM observation. The internal structure of the samples was observed using a transmission electron microscope (HT7700; Hitachi, Ltd.) (21). Subsequently, minute quantities of the samples were fixed on the copper network for characterization.

FT-IR study. The chemical bonding and functional groups of the samples were examined using an FT-IR spectrometer (Nicolet 380; Thermo Fisher Scientific, Inc., Waltham, MA, USA) (22). The samples were mixed with potassium bromide with a 1-2:200 ratio. Then, the mixture was pressed into a round cake and characterized in the scanning range of $400-3,500 \mathrm{~cm}^{-1}$.

Raman spectroscopy analysis. Raman spectroscopy (Thermo U-LH100L-3; Thermo Fisher Scientific, Inc.) was employed to examine the functional groups of the samples. After the slides were wrapped with tin foil, the samples were coated in foil. The samples were characterized in the scanning range of $50-3,300 \mathrm{~cm}^{-1}$, and scanning was concluded using the mode of area sweep.

Nitrogen adsorption analysis. Nitrogen adsorption-desorption analysis was performed using an adsorption analyzer (SA3100; Beckman Coulter, Inc., Brea, CA, USA). The physically adsorbed water of samples was removed by degasification at $50^{\circ} \mathrm{C}$ for $24 \mathrm{~h}$. Then, the container carrying the sample was immersed in liquid nitrogen. Subsequently, the specific surface area and the pore size of the samples were determined using the surface area analyzer.

$X R D$ and DSC analysis. The crystalline characteristics of samples were analyzed using an X-ray diffractometer (PW3040/60 PANALYII CALB.V, Almelo, The Netherlands) and the data were recorded (27). The scanned angle range of XRD patterns was $10^{\circ}-50^{\circ}$. DSC analysis was performed using a differential scanning calorimeter (HCR-1; Beijing Hengjiu Experimental Equipment Co., Ltd., Beijing, China). The temperature range for the DSC analysis was $50-200^{\circ} \mathrm{C}$ with a heating rate of $10^{\circ} \mathrm{C} / \mathrm{min}$.

In vitro drug release study. Dissolution experiments were conducted using a USP II paddle method with a dissolution instrument (RCZ-8B; Shanghai Huanghai Drug Testing Instrument Co., Ltd., Shanghai, China). Hydrochloric acid solution (pH 1.2) and phosphate buffer ( $\mathrm{pH}$ 6.8) were employed as dissolution media. The dissolution operation was as follows: dissolution media $(900 \mathrm{ml})$ was added to the basket and maintained at $37^{\circ} \mathrm{C}$. The added samples were stirred for $1 \mathrm{~h}$ at a rate of $100 \mathrm{rpm}$. In addition, $5 \mathrm{ml}$ samples were extracted at fixed time intervals $(5,10,15,20,30,45$ and $60 \mathrm{~min})$. Subsequently, an ultraviolet spectrophotometer (UV-2000; Unico, Franksville, WI, USA) at an excitation wavelength of $283 \mathrm{~nm}$ $(n=6)$ was used to determine the amount of DDM obtained from the samples. All measurements were repeated 6 times. The measurement data of the dissolution were expressed as the mean \pm standard deviation, $\mathrm{n}=6$.

\section{Results}

SEM observation. DDM was effectively loaded into MWCNTs using the solvent deposition method, which is a suitable method for loading drugs into MWCNTs $(29,30)$. It is well documented that long narrow channels play an important role in the release of drugs from MWCNTs $(31,32)$. SEM was employed to characterize the external morphology of the samples. As shown in Fig. 1A, the raw drug substance was observed to be strip-shaped and had no fixed length. MWCNTs were tubular in shape with a three-dimensional structure (Fig. 1B). The diameter of the MWCNTs was determined to be $<50 \mathrm{~nm}$ and MWCNTs were longer than $500 \mathrm{~nm}$. The cross-sectional width of MWCNTs was $\sim 25 \mathrm{~nm}$ and the pore diameter of MWCNTs was $\sim 2-10 \mathrm{~nm}$ (Figs. 1B and 2C). Fig. 1C demonstrates that MWCNTs surrounded the raw drug substance. This could be attributed to the fact that the raw drug was left untreated, and was directly mixed with the MWCNTs. In addition, the drug had not been loaded into the channels of the MWCNTs. It was apparent that MWCNTs surrounded the raw drug substance. After the DDM was incorporated into MWCNTs using the solvent deposition method, the bulk of the raw drug substance could not be found, indicating that a large number of raw drugs were loaded into the channels of the MWCNTs or attached to the surface of MWCNTs. Fig. 1D presents the SEM image of DM-25. Also, the size of the occupying space was observed to be not regular because of the random distribution of the drug inside or on the surface of the channels. 

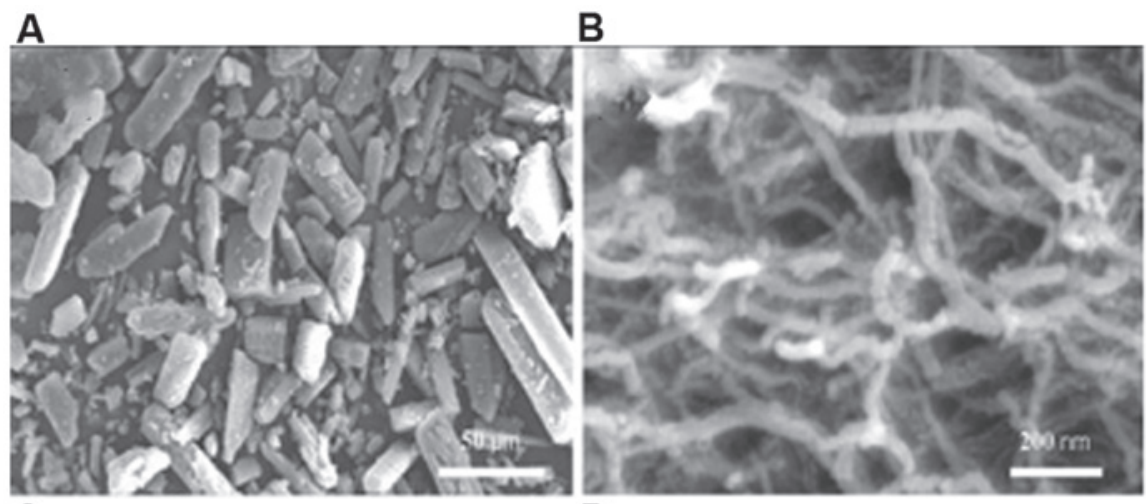

C

D

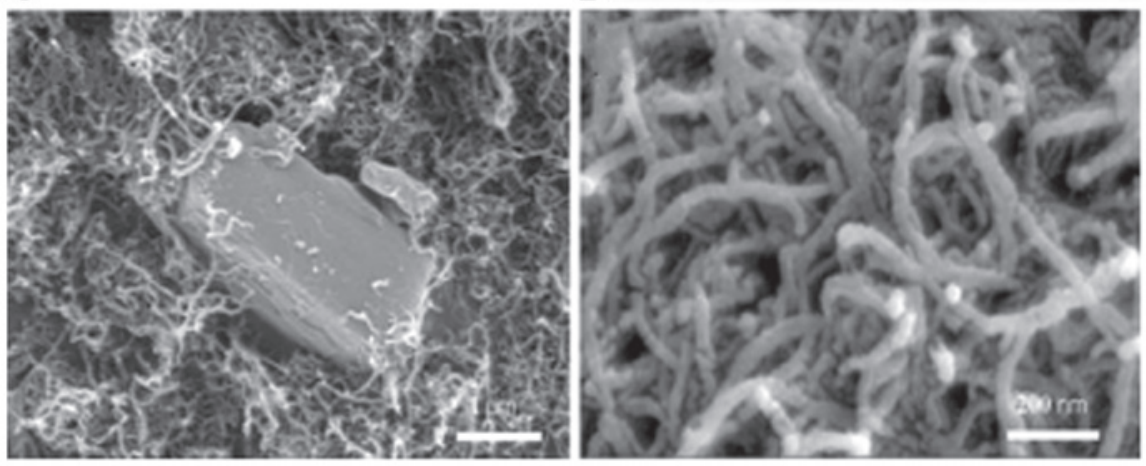

Figure 1. SEM images of (A) DDM, (B) MWCNTs, (C) DMP and (D) DM-25. SEM, scanning electron microscopy; DDM, dipyridamole; MWCNTs, multi-walled carbon nanotubes; DMP, MWCNT-DDM physical mixture.

A

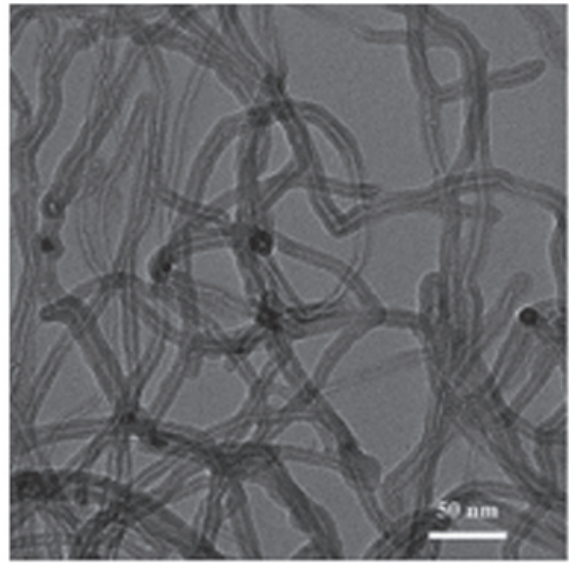

C

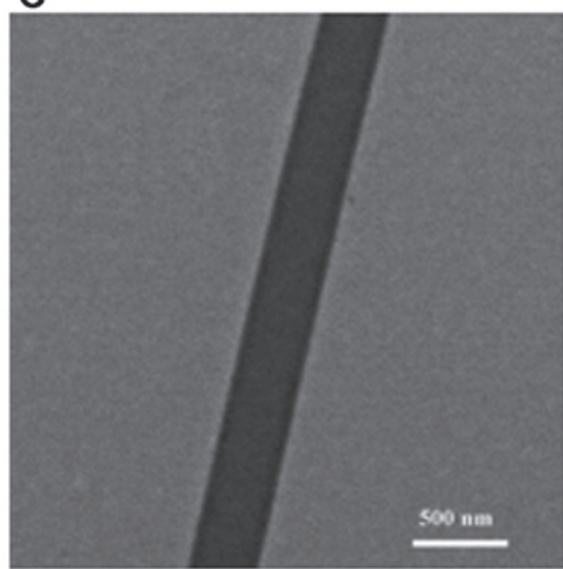

B

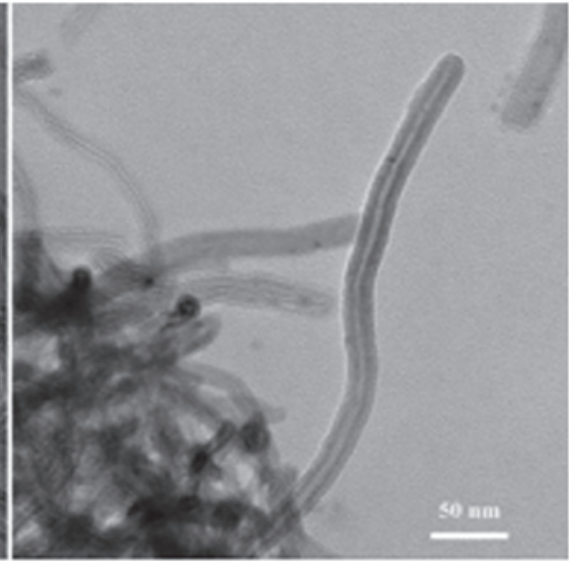

D

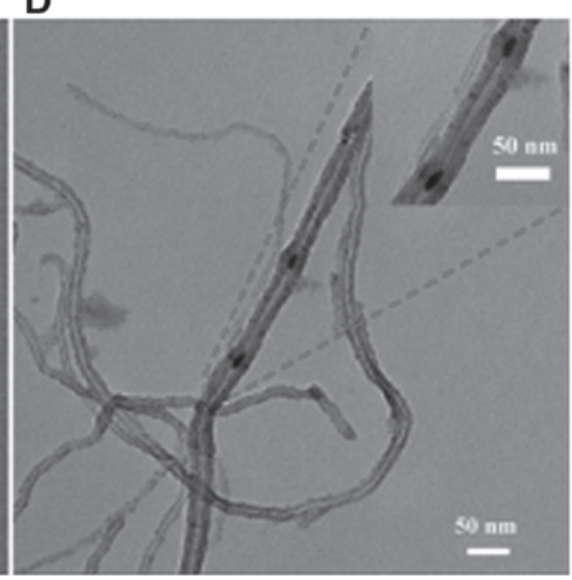

Figure 2. TEM images of (A) MWCNTs (state of aggregation), (B) MWCNTs (single), (C) MWCNT (amplification) and (D) DM-25. TEM, transmission electron microscopy; MWCNTs, multi-walled carbon nanotubes. 


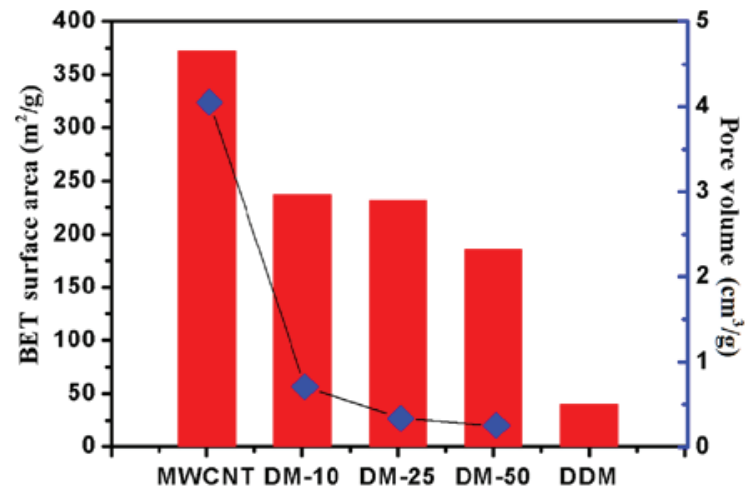

Figure 3. Specific surface area and pore volume of MWCNT, DM-10, DM-25, DM-50 and DDM. MWCNT, multi-walled carbon nanotube; DDM, dipyridamole.

TEM observation. TEM was applied to identify the morphology of the samples. As shown in Fig. 2A and B, the long tubular structures of MWCNTs were visible and intertwined with each other. The tube wall structure was visible in the cross-section of the MWCNTs. The channels were sandwiched between the long and narrow pipe walls of the MWCNTs. This specific structure of the MWCNTs played important role in the release of drugs. The channel structure of a MWCNT is enlarged in Fig. $2 \mathrm{C}$ in order to observe the channel structure distinctly. It is clearly revealed that the inner cavity of the tunnel was hollow, which could potentially be used for drug loading. As seen in Fig. 2D, in contrast to unloaded MWCNTs, the channels of the MWCNTs samples were blocked by some particles, indicating that drugs were loaded into the channels of the MWCNTs.

Nitrogen adsorption analysis. In the current study we further assessed the progress of drug loading using nitrogen adsorption measurements. As shown in Fig. 3, the pore volume and the surface area of DM-10, DM-25 and DM-50 were reduced compared with that of the MWCNTs (unloaded drugs).

FT-IR analysis. FT-IR method was used to further confirm the progress of loading DDM into the channel of MWCNT. As shown in Fig. 4D, DDM presented a characteristic peak at $\sim 3,500-3,000 \mathrm{~cm}^{-1}$, which reflects the characteristics of hydroxyl peaks. The existence of the hydroxyl peak was also supported by the vibration peak at $1,500-1,260 \mathrm{~cm}^{-1}$. In addition, the MWCNT exhibited peaks at 1,600-1,700 $\mathrm{cm}^{-1}$, which corresponds to the $\mathrm{C}=\mathrm{C}$ expansion vibration absorption peaks (Fig. 4F). This absorption peak arose from the skeleton of MWCNT. After the completion of the drug-loading progress, the enol-type structure is possible to be formed as a result of the association between the $\mathrm{C}=\mathrm{C}$ double bond with the hydroxyl group. Therefore, as expected, DM-10, DM-25 and DM-50 showed the characteristic peaks of the enol structure at $\sim 1,700 \mathrm{~cm}^{-1}$ (Fig. 4A-C). The characteristic peak was not obvious, which may be attributed to the instability of the enol-type structure.

Raman spectrum analysis. Raman spectra proved to be very beneficial for monitoring the drug-loading process. As shown in Fig. 5E, the Raman spectra had a main band with

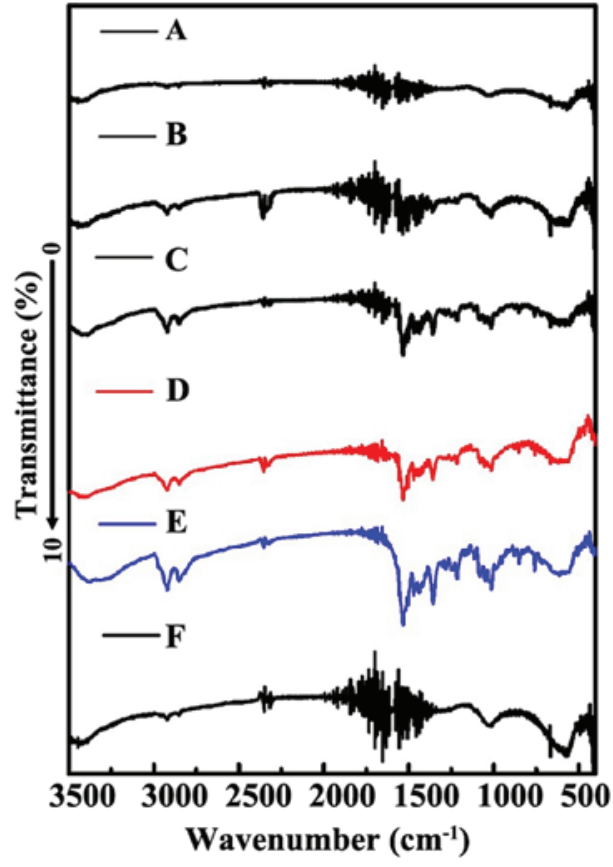

Figure 4. FT-IR spectra for (A) DM-10, (B) DM-25, (C) DM-50, (D) DMP, (E) DDM and (F) MWCNT. FT-IR, Fourier transform-infrared; DMP, MWCNT-DDM physical mixture; DDM, dipyridamole; MWCNT, multi-walled carbon nanotube.

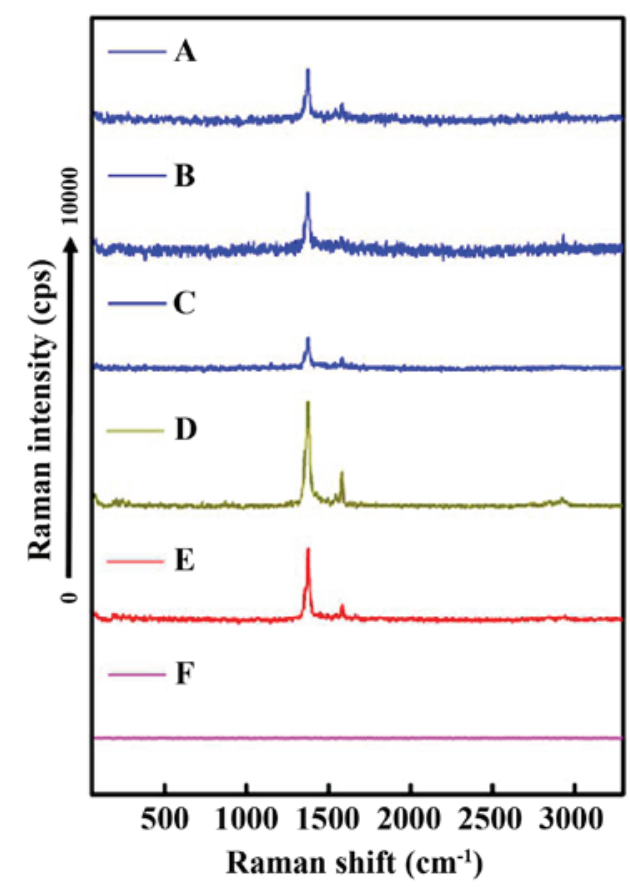

Figure 5. Raman spectra for (A) DM-50, (B) DM-25, (C) DM-10, (D) DMP, (E) DDM and (F) MWCNT. DMP, MWCNT-DDM physical mixture; DDM, dipyridamole; MWCNT, multi-walled carbon nanotube.

a maximum at $1,375 \mathrm{~cm}^{-1}$ and a low frequency band with a maximum near $1,580 \mathrm{~cm}^{-1}$. For DM-10, DM-25 and DM-50, the characteristic peaks of the DDM were apparent for all, indicating the existence of DDM in the MWCNT-DDM delivery system. Moreover, the results illustrated that DDM was successfully loaded into the MWCNTs. The MWCNT exhibited no peaks in the range of $50-3,300 \mathrm{~cm}^{-1}$ (Fig. 5F). The 


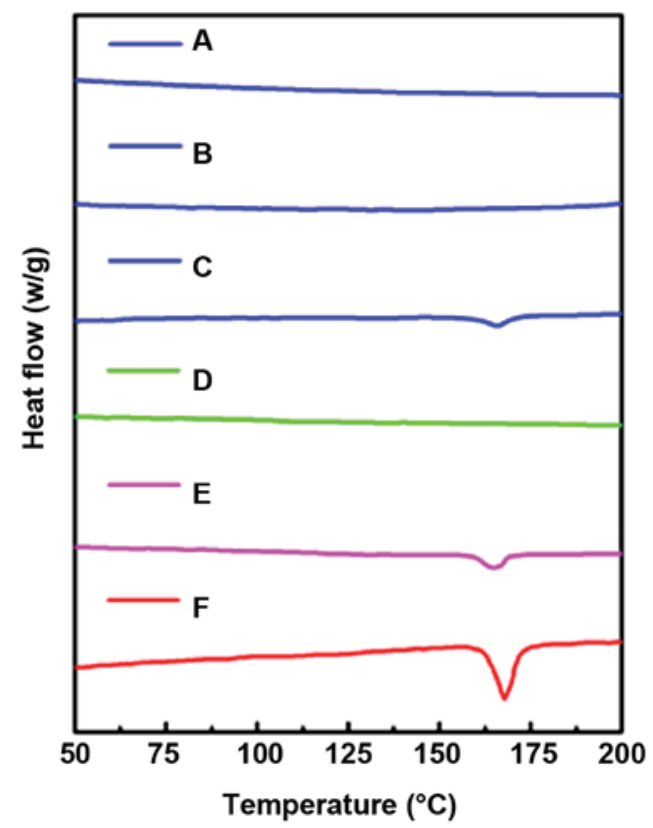

Figure 6. DSC patterns of (A) DM-10, (B) DM-25, (C) DM-50, (D) MWCNT, (E) DMP and (F) DDM. DSC, differential scanning calorimetry; MWCNT, multi-walled carbon nanotube; DMP, MWCNT-DDM physical mixture; DDM, dipyridamole.

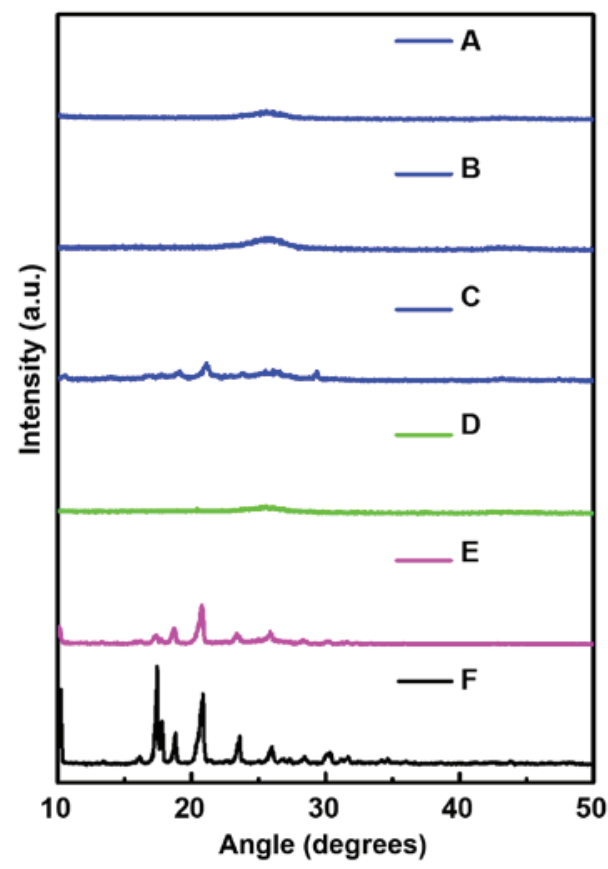

Figure 7. XRD patterns of (A) DM-10, (B) DM-25, (C) DM-50, (D) MWCNT, (E) DMP and (F) DDM. XRD, X-ray diffraction; MWCNT, multi-walled carbon nanotube; DMP, MWCNT-DDM physical mixture; DDM, dipyridamole.

characteristic peaks of MWCNT with DDM (DM-10, DM-25 and DM-50) were observed at 1,375 and $1,580 \mathrm{~cm}^{-1}$.

Solid state study by DSC and XRD. DSC was employed to confirm the crystal characteristics of the samples (28). As shown in Fig. 6, the strong endothermic melting peak was observed at $165.6^{\circ} \mathrm{C}$, which is the melting point of DDM. Conversely,

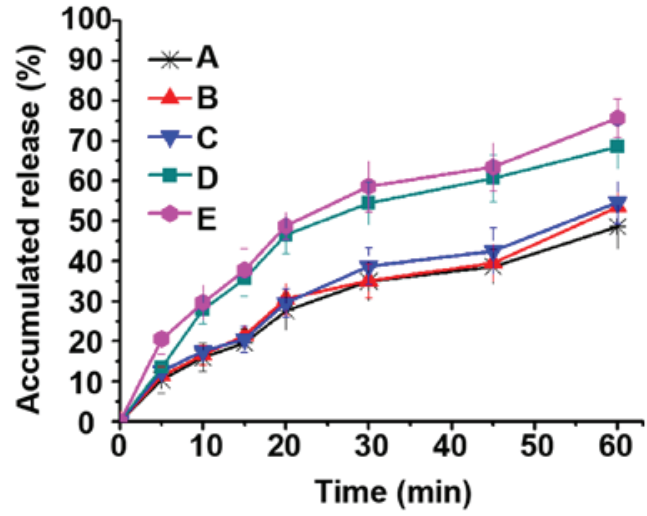

Figure 8. Dissolution profiles of (A) DDM, (B) DMP, (C) DM-50, (D) DM-25 and (E) DM-10 in acid medium (pH 1.5). DDM, dipyridamole; DMP, MWCNT-DDM physical mixture.

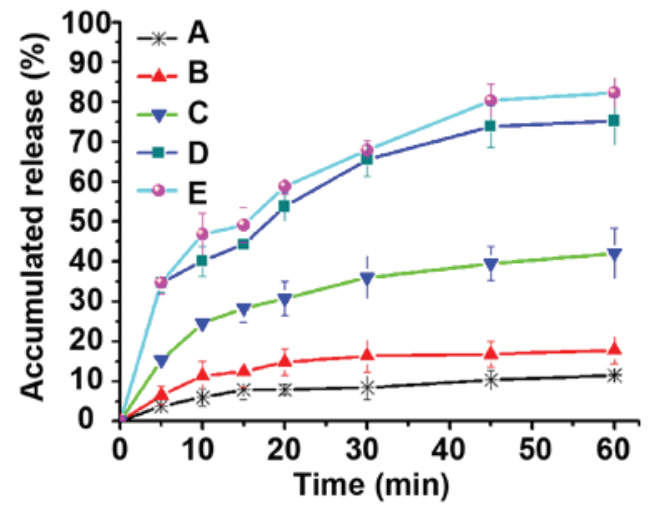

Figure 9. Dissolution profiles of (A) DDM, (B) DMP, (C) DM-50, (D) DM-25 and (E) DM-10 in weak-basic medium (pH 6.8). DDM, dipyridamole; DMP, MWCNT-DDM physical mixture.

MWCNT did not exhibit any peak at the corresponding temperature. The endothermic melting peak of DM-50 was still present. However, the inverted peak of DM-25 and DM-50 were not observed at the corresponding melting point.

XRD study was used to evaluate the crystal properties of the samples with a fixed angle ranging from $10^{\circ}$ to $50^{\circ}$. As shown in Fig. 7, three intense and characteristic peaks of pure DDM were observed at $17.4^{\circ}, 17.8^{\circ}$ and $20.8^{\circ}$. However, the same peaks were not found in the corresponding position for MWCNT. In fact, a completely different diffraction peak at $24.7^{\circ}$ was noted for MWCNT. When the DDM was physically mixed with MWCNT, the diffraction peak of the DDM was found to be slightly reduced.

In vitro drug dissolution. Dissolution experiments were performed in order to explore the application of MWCNTs as carriers for DDM and to elucidate the effect of different drug-loading rates on the dissolution behavior of DDM. As shown in Fig. 8, the $1 \mathrm{~h}$ cumulative release of DDM was found to be $\sim 50 \%$ in acid medium ( $\mathrm{pH} 1.5$ ). However, the dissolution rates of DM-10 and DM-25 were observed to be higher than DM-50. The results revealed that the dissolution behavior of the DM-50 curve was very close to that of the DDM in acidic medium (pH 1.5), indicating that the effect of the MWCNT on DDM dissolution behavior was not significant. Notably, it showed good 
solubility in acidic medium due to the chemical nature of DDM. DDM is easily soluble in acidic medium ( $\mathrm{pH} 1.5)$ because of its chemical properties. So, the $1 \mathrm{~h}$ cumulative release of DDM was found to be $\sim 50 \%$ in acidic medium ( $\mathrm{pH} 1.5$ ).

As shown in Fig. 9, the cumulative release amount of DDM was just $10 \%$ in weak-basic medium ( $\mathrm{pH} 6.8$ ), while the cumulative release amounts of DM-10, DM-25 and DM-50 were all especially higher than that of the DDM. Moreover, some drugs on the surface of the DM-50 showed a similar release behavior to that of DDM, which is also consistent with the results of DSC and XRD. Interestingly, both DM-50 and DDM showed the same crystalline characteristics. This could be explained by the fact that some drugs of DM-50 might have been gathered on the surface of the MWCNTs, leading to similar dissolution behavior as pure DDM. However, the dissolution rates of DM-10 and DM-25 were higher than that of DM-50 in acidic medium because DM-10 and DM-25 drugs were primarily localized in the interior of the MWCNTs, resulting in relatively more uniform distribution (Figs. 8 and 9). This particular finding demonstrated that MWCNTs covered up the crystalline form of DDM when drug loading was 10 and $25 \%$. Furthermore, MWCNTs did not entirely change the crystalline form of DDM into an amorphous form when the drug loading was up to $50 \%$. In the weak-basic medium ( $\mathrm{pH} 6.8$, Fig. 9), the cumulative release amount of DDM was found to be merely $10 \%$, which was attributable to DDM that is a BCS class II drug and has poor dissolution. However, the cumulative release amounts of DM-10, DM-25 and DM-50 were all especially higher than that of the DDM. This suggested that MWCNTs had improved the dissolution of crude DDM as a result of dispersion of the drug. In addition, MWCNTs limited the crystal growth of the DDM. So, it could be inferred that the MWCNTs reduced the degree of DDM crystallization or maintained DDM in a non-crystalline state. It is well known that the drag solubility in a non-crystalline state is higher than that in the crystalline state (33-35). The results of DSC and XRD showed that the crystallinities of DM-10, DM-25 and DM-50 were all decreased, and even became amorphous, in contrast to that of DDM. Moreover, the dissolution behavior of the samples was also found to be affected by the drug-loading rate. Increasing the drug-loading rate led to slight decrease in the dissolution rate of samples. DDM in the DM-10 and DM-25 was in a non-crystalline state. In contrast, DDM in the DM-50 was in the original crystalline state. This may be due to the drugs being completely loaded into the channels of the MWCNT, when drug-loading rates were relatively low (10 and 25\%). Accordingly, DM-10 and DM-25 were both in amorphous forms. However, drugs may not have been completely loaded into the channels of the MWCNTs and some drugs were still deposited on the surface of the MWCNT, when drug loading was relatively higher (50\%). This was also the reason why the drugs in the DM-50 were in crystalline form and the crystalline form was different from the DM-10 and DM-25. Moreover, the dissolution rate of DM-10 was found to be a little higher than that of DM-25, whereas the dissolution rate of DM-50 was slower than that of DM-10 and DM-25. These findings were highly suggestive that the dissolution rate of DDM-MWCNT system decreased upon increasing the drug-loading rate. The drugs were in different positions of the MWCNTs for different drug-loading rates, which led to different crystalline forms of the drugs. This particular finding is also in line with the results of DSC. The results of DSC showed that the drugs in the DM-50 were in crystal state, but the drugs in the DM-10 and DM-25 were amorphous, explaining that the solubility of drugs was affected by the crystalline form. Similarly, it has been reported that the solubility of the crystal drugs is lower than that of the amorphous drugs $(36,37)$. To further explore the causes, the drug-loading rate determined the crystal form of drugs in the MWCNT system, and also affected their dissolution rates. Drug loading is a key factor in the MWCNT system, which may affect the crystal morphology of drugs and the dissolution of drugs. Therefore, the aforementioned results indicate that the drug dissolution rate in MWCNTs is directly affected by the drug-loading rate.

\section{Discussion}

Studies have shown that MWCNT is a suitable carrier for poorly soluble drugs $(24,38)$. On the one hand, the current study developed the application range of MWCNTs in the field of pharmacy, on the other hand, it attempted to develop a new platform for the delivery of poorly soluble drugs. In the present study MWCNTs were presented to effectively solve the problem of low solubility of DDM. The improvement of the dissolution rate is of great significance for the oral absorption of DDM. The screening of drug-loading methods for MWCNTs and the optimization of drug-loading processes are not perfect. MWCNTs as drug carriers still have some limitations. There are many problems, such as poor controllability of the length of the pipe and the intertwining of the pipes, that affect the drug-loading processes. The effect of the carrier structure on the drug-loading processes will be the aim of our future research.

\section{Acknowledgements}

Not applicable.

\section{Funding}

This study was supported by the Science and Technology General Program of Heilongjiang Educational Committee (no. 2016-KYYWF-0857).

\section{Availability of data and materials}

The datasets used and/or analyzed during the present study are available from the corresponding author on reasonable request.

\section{Authors' contributions}

WZ drafted the manuscript. WZ and $\mathrm{HH}$ were mainly devoted to the synthesis of the MWCNT-DDM system. YD and $\mathrm{CH}$ assisted with the characterization techniques. XS and BJ were responsible for the in vitro drug release study. All the authors read and approved the final manuscript.

\section{Ethics approval and consent to participate}

Not applicable. 


\section{Patient consent for publication}

Not applicable.

\section{Competing interests}

The authors declare that they have no competing interests.

\section{References}

1. D'Souza SL, Deshmukh B, Bhamore JR, Rawat KA, Lenka N and Kailasa SK: Synthesis of fluorescent nitrogen-doped carbon dots from dried shrimps for cell imaging and boldine drug delivery system. Rsc Adv 6: 12169-12179, 2016.

2. Zhang H, Hou L, Jiao X, Ji Y, Zhu X and Zhang Z: Transferrin-mediated fullerenes nanoparticles as $\mathrm{Fe}(2+)$-dependent drug vehicles for synergistic anti-tumor efficacy. Biomaterials 37 : 353-366, 2015.

3. Wang Q, Huang X, Long Y, Wang X, Zhang H, Zhu R, Liang L, Teng $\mathrm{P}$ and Zheng $\mathrm{H}$ : Hollow luminescent carbon dots for drug delivery. Carbon 59: 192-199, 2013.

4. Potter C, Tian Y, Walker G, McCoy C, Hornsby P, Donnelly C, Jones DS and Andrews GP: Novel supercritical carbon dioxide impregnation technique for the production of amorphous solid drug dispersions: A comparison to hot melt extrusion. Mol Pharm 12: 1377-1390, 2015

5. Muzi L, Ménard-Moyon C, Russier J, Li J, Chin CF, Ang WH, Pastorin G, Risuleo G and Bianco A: Diameter-dependent release of a cisplatin pro-drug from small and large functionalized carbon nanotubes. Nanoscale 7: 5383-5394, 2015.

6. Yao MZ, Huang-Fu MY, Liu HN, Wang XR, Sheng X and Gao JQ Fabrication and characterization of drug-loaded nano-hydroxyapatite/polyamide 66 scaffolds modified with carbon nanotubes and silk fibroin. Int J Nanomedicine 11: 6181-6194, 2016.

7. Sciortino N, Fedeli S, Paoli P, Brandi A, Chiarugi P, Severi M and Cicchi S: Multiwalled carbon nanotubes for drug delivery: Efficiency related to length and incubation time. Int J Pharm 521: 69-72, 2017.

8. Bhirde AA, Patel V, Gavard J,Zhang G, Sousa AA, Masedunskas A, Leapman RD, Weigert R, Gutkind JS and Rusling JF: Targeted killing of cancer cells in vivo and in vitro with EGF-directed carbon nanotube-based drug delivery. ACS Nano 3: 307-316, 2009

9. Ajima K, Yudasaka M, Murakami T, Maigné A, Shiba K and Iijima S: Carbon nanohorns as anticancer drug carriers. Mol Pharm 2: 475-480, 2005.

10. Heister E, Neves V, Lamprecht C, Silva SRP, Coley HM and McFadden J: Drug loading, dispersion stability, and therapeutic efficacy in targeted drug delivery with carbon nanotubes. Carbon 50: 622-632, 2012.

11. Cheng Y, Lu S, Zhang H, Varanasi CV and Liu J: Synergistic effects from graphene and carbon nanotubes enable flexible and robust electrodes for high-performance supercapacitors. Nano Lett 12: 4206-4211, 2012

12. Yuan L, Lu XH, Xiao X, Zhai T, Dai J, Zhang F, Hu B, Wang X, Gong L, Chen J, et al: Flexible solid-state supercapacitors based on carbon nanoparticles $/ \mathrm{MnO}_{2}$ nanorods hybrid structure. ACS Nano 6: 656-661, 2012.

13. Park H, Afzali A, Han SJ, Tulevski GS, Franklin AD, Tersoff J, Hannon JB and Haensch W: High-density integration of carbon nanotubes via chemical self-assembly. Nat Nanotechnol 7: 787-791, 2012

14. Ncibi MC and Sillanpää M: Optimizing the removal of pharmaceutical drugs Carbamazepine and Dorzolamide from aqueous solutions using mesoporous activated carbons and multi-walled carbon nanotubes. J Mol Liq 238: 379-388, 2017.

15. Lee G, Choong H, Chiang G and Woo K: Three-year randomized controlled trial of dipyridamole and low-dose warfarin in patients with $\operatorname{Ig}$ A nephropathy and renal impairment. Nephrology (Carlton) 3: 117-121, 2010

16. Tang Y, Liu SY, Armes SP and Billingham NC: Solubilization and controlled release of a hydrophobic drug using novel micelleforming $\mathrm{ABC}$ triblock copolymers. Biomacromolecules 4: $1636-1645,2003$

17. de Waard H, Hessels MJT, Boon M, Sjollema KA, Hinrichs WLJ, Eissens AC and Frijlink HW: CLSM as quantitative method to determine the size of drug crystals in a solid dispersion. Pharm Res 28: 2567-2574, 2011.
18. Zecevic DE, Meier R, Daniels R and Wagner KG: Site specific solubility improvement using solid dispersions of HPMC-AS/HPC SSL-mixtures. Eur J Pharm Biopharm 87: 264-270, 2014.

19. Sakai $T$ and Thommes M: Investigation into mixing capability and solid dispersion preparation using the DSM Xplore Pharma Micro Extruder. J Pharm Pharmacol 66: 218-231, 2014.

20. Zhao L, Gray L, Leonardi-Bee J, Weaver CS, Heptinstall S and Bath PM: Effect of aspirin, clopidogrel and dipyridamole on soluble markers of vascular function in normal volunteers and patients with prior ischaemic stroke. Platelets 17: 100-104, 2006.

21. Yan YD, Sung JH, Kim KK, Kim DW, Kim JO, Lee BJ, Yong CS and Choi HG: Novel valsartan-loaded solid dispersion with enhanced bioavailability and no crystalline changes. Int J Pharm 422: 202-210, 2012.

22. Mahmoodi M, Arjmand M, Sundararaj U and Park S: The electrical conductivity and electromagnetic interference shielding of injection molded multi-walled carbon nanotube/polystyrene composites. Carbon 50: 1455-1464, 2012.

23. Gupta TK, Singh BP, Mathur RB and Dhakate SR: Multi-walled carbon nanotube-graphene-polyaniline multiphase nanocomposite with superior electromagnetic shielding effectiveness. Nanoscale 6: 842-851, 2014.

24. Pippa N, Chronopoulos DD, Stellas D, Fernández-Pacheco R, Arenal R, Demetzos C and Tagmatarchis N: Design and development of multi-walled carbon nanotube-liposome drug delivery platforms. Int J Pharm 528: 429-439, 2017.

25. Bottini M, Bruckner S, Nika K, Bottini N, Bellucci S, Magrini A, Bergamaschi A and Mustelin T: Multi-walled carbon nanotubes induce T lymphocyte apoptosis. Toxicol Lett 160: 121-126, 2006.

26. Gabizon AA, Barenholz Y and Bialer M: Prolongation of the circulation time of doxorubicin encapsulated in liposomes containing a polyethylene glycol-derivatized phospholipid: Pharmacokinetic studies in rodents and dogs. Pharm Res 10: 703-708, 1993

27. Zhu W, Zhao Q, Sun C, Zhang Z, Jiang T, Sun J, Li Y and Wang S: Mesoporous carbon with spherical pores as a carrier for celecoxib with needle-like crystallinity: Improve dissolution rate and bioavailability. Mater Sci Eng C 39: 13-20, 2014.

28. Zhu WQ, Wan L, Zhang C, Gao YK, Zheng X, Jiang TY and Wang SL: Exploitation of 3D face-centered cubic mesoporous silica as a carrier for a poorly water soluble drug: Influence of pore size on release rate. Mater Sci Eng C 34: 78-85, 2014.

29. Zhang X, Hui Z, Wan D, Huang H, Huang J, Yuan H and Yu J: Alginate microsphere filled with carbon nanotube as drug carrier. Int J Biol Macromol 47: 389-395, 2010.

30. Shao W, Paul A, Zhao B, Lee C, Rodes L and Prakash S: Carbon nanotube lipid drug approach for targeted delivery of a chemotherapy drug in a human breast cancer xenograft animal model. Biomaterials 34: 10109-10119, 2013.

31. Wong BS, Yoong SL, Jagusiak A, Panczyk T, Ho HK, Ang WH and Pastorin G: Carbon nanotubes for delivery of small molecule drugs. Adv Drug Deliv Rev 65: 1964-2015, 2013.

32. Fan J, Zeng F, Xu J and Wu S: Targeted anti-cancer prodrug based on carbon nanotube with photodynamic therapeutic effect and pH-triggered drug release. J Nanopart Res 15: 1911, 2013.

33. Chen J, Sarma B, Evans JMB and Myerson AS: Pharmaceutical crystallization. Cryst Growth Des 11: 887-895, 2011.

34. Weimann PA, Hajduk DA, Chu C, Chaffin KA, Brodil JC and Bates FS: Crystallization of tethered polyethylene in confined geometries. J Polym Sci Pol Phys37: 2053-2068, 2015.

35. Leon RAL, Wan WY, Badruddoza AZM, Hatton TA and Khan SA: Simultaneous spherical crystallization and co-formulation of $\operatorname{drug}(\mathrm{s})$ and excipient from microfluidic double emulsions. Cryst Growth Des 14: 140-146, 2014.

36. Löbmann K, Laitinen R, Grohganz H, Gordon KC, Strachan C and Rades T: Coamorphous drug systems: Enhanced physical stability and dissolution rate of indomethacin and naproxen. Mol Pharm 8: 1919-1928, 2011.

37. Shegokar R and Müller RH: Nanocrystals: Industrially feasible multifunctional formulation technology for poorly soluble actives. Int J Pharm 399: 129-139, 2010.

38. Ezzati Nazhad Dolatabadi J, Omidi Y and Losic D: Carbon nanotubes as an advanced drug and gene delivery nanosystem. Curr Nanosci 7: 297-314, 2011.

This work is licensed under a Creative Commons Attribution-NonCommercial-NoDerivatives 4.0 International (CC BY-NC-ND 4.0) License. 